Ocean. It is likely that when snow particles collide, the particles acquire a negative charge, whilst a positive charge is given to the air. The snow falls to the ground and a positive space charge remains in the air and may extend to a considerable height. The process is analogous to what happens in a sandstorm, though the sign of the electrification is reversed. Thus it is to be expected that in the part of a cloud where there are snow particles in violent motion there will be electrification, that the snow will carry down a negative charge and that the air streaming upwards will take a positive charge to the top of the cloud, this charge being communicated rapidly to the cloud particles. It may be added that as the cloud-top is in a region where the conductivity is high, a strong current of positive electricity can flow upwards to the Heaviside layer, so providing (in accordance with Wilson's theory) for the air-earth current of fine weather.

There is some evidence that the regions in which positive electrification is produced low down in the clouds are those where the rain is heaviest, and Simpson and Scrase hold that the breaking-drop theory of the former is the explanation of this electrification. As the snow melts, it forms large drops, and if these are too large for stability they will break up. The rising current of air will carry a negative charge and the positively charged droplets fall or perhaps remain nearly stationary. The hypothesis that this process occurs only where the upward current is strong is embodied in Fig. 2.

Perhaps the most surprising result of the investigation is that no evidence is found for very strong fields in the clouds. The magnitude of the potential gradient is determined by a tentative formula which takes account of the width of the trace on the electrogram and also of the density of the air. It was found that when the gradient exceeded about 100 volts per centimetre, the trace was marked by sparking, but this occurred in only a few of the ascents. In 1925 Wilson put forward the hypothesis that, when a lightning flash is about to occur, the electric field just below a thundercloud is probably nearly 10,000 volts per centimetre, a hundred times the strength near the ground. The hypothesis seemed to be supported by estimates of the space charge produced by point discharge from trees and other objects. The soundings indicate, however, that generally there is little if any increase in the gradient as the cloud is approached. It may be that the estimates are systematically in error, and it is to be hoped that control measurements will be made by some more direct method.

One reason for suspecting such systematic error is that the estimated gradients imply that the charges in the clouds are comparatively small. If the gradients over two planes a kilometre apart are $+100 \mathrm{v} . / \mathrm{cm}$. and $-100 \mathrm{v} . / \mathrm{cm}$. respectively the charge is (by Coulomb's law) only 0.2 coulombs per cubic kilometre. This is insignificant compared with 20 coulombs, the quantity of electricity supposed to be discharged by a lightning flash. In what part of a cloud the lightning originates, and why it occurs, we have yet to discover.

The investigation is being continued at Kew and, although the ascents made during 1937 have not led to any striking new discoveries, it may be that in the course of time a record will be obtained which will provide the missing clue.

F. J. W. WHIPPLE.

\title{
Heat Production, Nutrition, and Growth in Man: Some New Views*
}

\author{
By Dr. E. P. Poulton
}

\section{INDIRECT AND DiRECT CALORIMETRY}

$\mathrm{I}^{\mathrm{N}}$ $\mathrm{N}$ estimating the heat production of an individual, the oxygen intake and the carbon dioxide output are measured. After allowing for protein metabolism the oxygen intake is converted into calories of heat generated, by multiplying it with a factor which depends on the respiratory quotient (volume of carbon dioxide produced/volume of oxygen absorbed). These multiplying factors were

- Substance of the presidential address delivered to Section I (Physiology) of the British Association at Nottingham on September 2, revised and modifled. calculated originally by Zuntz and Schumburg (1901), and are based upon: (a) the heats of combustion of carbohydrate and fat; $(b)$ the chemical composition of carbohydrate and fat; and $(c)$ the theory that when the respiratory quotient has the value unity carbohydrate alone is oxidized in the body and when the respiratory quotient has a value equal to 0.707 fat only is being oxidized, and at intermediate values of the respiratory quotient corresponding proportions of carbohydrate and fat are being oxidized. This method of calculating the heat produced by an individual is known as 
indirect calorimetry. The basal metabolism or basal metabolic rate of American authors or 'standard' metabolism of Krogh and Lindhard is at present defined as heat production in the morning before breakfast, fourteen to sixteen hours after the last meal of the previous day (the postabsorptive condition) with the subject lying at rest.

The heat output of man and animals, under basal conditions, has been measured directly in a calorimeter by Benedict and Carpenter, DuBois and his colleagues and Murlin and Lusk, and compared with the heat output calculated indirectly by means of the Zuntz-Schumburg figures from the oxygen intake and the carbon dioxide output, which were measured simultaneously. The results obtained have been held by their authors to justify

(direct heat). The difference between the indirect and direct heat was expressed as a percentage of the direct heat and related to the respiratory quotient by a graphic method. The result is shown in Figs. $1 a$ and $1 b$. The horizontal line at zero in the top part of the diagrams represents the condition where the indirect and direct heats are identical; the ordinates represent the per: centage differences between the indirect and direct heats. Satisfactory agreement between the heat calculated and the direct heat will depend on the percentage differences, indicated by the black dots, being evenly distributed above and below the horizontal zero line throughout the range of respiratory quotients. This is obviously not the case. The positions of the points suggest a systematic error ; for the lower quotients the indirect heat is too high and for the higher quotients too low. DuBois' results tend to show the same thing, though not in such a striking way.

Another way of studying this question is to plot the carbon dioxide and oxygen independently against the heat (Fig. 2). The position of the points in the diagrams shows that there is a linear relation between the carbon dioxide and calories (correlation coefficient $0.950 \pm 0.005)$ and between oxygen and calories (correlation coefficient $0.92 \pm$ 0.008 ). The carbohydrate line is the relation of the carbon dioxide output and oxygen intake to the heat produced, when pure carbohydrate is burnt in a calorimeter ; they were calculated from the value given by Lusk, namely, $5 \cdot 047$ Cal. per litre of oxygen and carbon dioxide. The lines $K$ and

Fig. $1 b$.

COMPARISON OF INDIRECT AND DIRECT CALORLMETRY. $0=$ OXYGEN $+=\mathrm{CO}$, ; TWO-HOURLY DETERMINATIONS DURING FIRST DAY OF FASTING. SUBJECT AT REST. IN Fig. I $a$ Two OBSERVATIONS COINCIDED AND SO ONLY 20 ARE SHOWN.

the conclusion from average values that the agreement between the two methods was sufficiently satisfactory to give support to the theory of the 'variable combustion ratio' outlined above. However, this theory has not escaped criticism.

\section{AN ApParent ERror in INDIRECT CALORIMETRY}

The most complete study of direct and indirect calorimetry in normal man under basal conditions is that made by Benedict and Carpenter. Using the data for two subjects, the theoretical heat from the oxygen intake and the respiratory quotient was calculated by the Zuntz-Schumburg method, and this heat (indirect heat) was compared with the heat actually measured by the calorimeter
$M$ represent the relation of the heat to the carbon dioxide output on the left-hand side and to the oxygen intake on the right when two different kinds of human fat are burnt. These two fats, from liver and skeletal muscle analysed by Cathcart and Cuthbertson, were chosen because they represent extremes, and the lines of the other nine human fats analysed by them lie in between. Most of the carbon dioxide points lie between the theoretical carbohydrate and fat lines, which are widely spaced; but this is certainly not the case with the oxygen points, as the theoretical limits are much narrower. It is possible to look on the carbon dioxide as the product of combustion of a mixture of carbohydrate and fat, but, because the oxygen points lie for the most part outside the theoretical limits, the oxygen intake cannot, 
apparently, be entirely used up in combustion, but must in part be concerned in some kind of conversion.

However, work since Nottingham has exposed an error in these diagrams. In many of DuBois' results there was a considerable difference in the rectal temperature at the beginning and end of an experiment which was taken to imply a large physical liberation or storage of heat, depending on the temperature difference, the body weight and average specific heat of the body $(0 \cdot 83)$. But since the rectal temperature can only be a rough measure of the body temperature as a whole, it follows that when there are large differences between the eliminated heat (actually measured) and the direct calorimetry (the value after correction for heat physically liberated or stored), there must be considerable uncertainty about the results. It has been found on plotting heat against carbon dioxide, according to the quotient, that when the differences are greater or less than 10 per cent of the eliminated heat, many points fall a long way from the mean line ; the eliminated heat has apparently been overcorrected. These uncertain results also form a high proportion of those outside the theoretical limits for oxygen and calories in Fig. 2, and they also seem to account for the similarity of the results of Benedict and DuBois, when the latter are calculated so as to form diagrams as in the upper part of Figs. $1 a$ and $1 b$. If all the uncertain results are eliminated the points in Fig. 2 lie nearer to the mean line, and now appear compatible with the theory of the variable combustion ratio. There is thus a
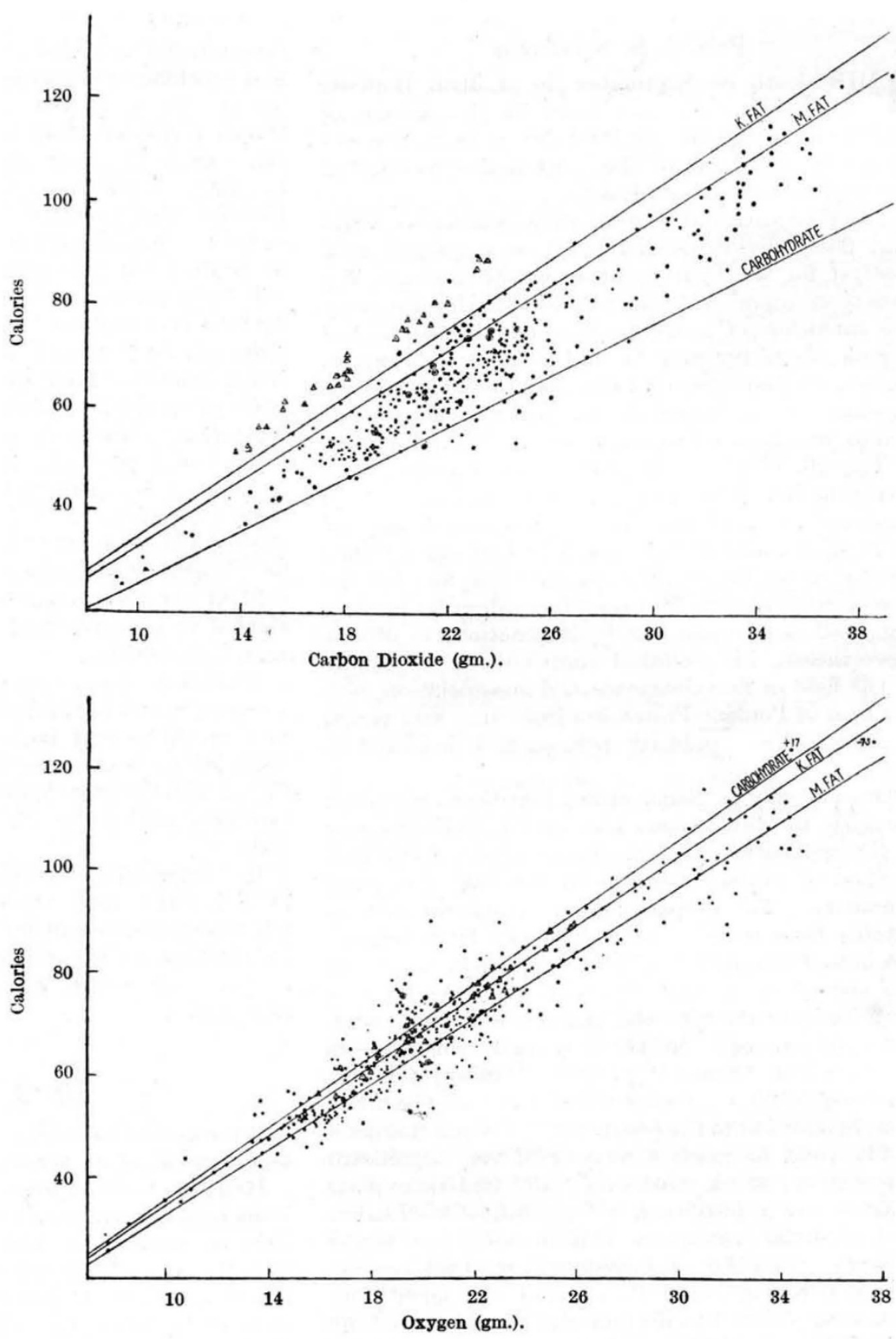

Fig. 2.

COMPARISON OF INDIRECT AND DIRECT CALORIMETRY. DUBOIS' BASAL VALUES (RECTAL TEMPERATURES NOT ABOVE $37.5^{\circ} \mathrm{C}$.). ORDinates : CALORIES PFR HOUR; ABSCISS 9 : UPPER DIAGRAM- $\mathrm{CO}_{2}$ GM. PER HOUR; Lower diagram $\mathrm{O}_{2}$ GM. per hour. $\odot$ Two coINCIDENT VALUes. $\triangle$ ALCOHOL CONTROL EXPERIMENTS.

discrepancy between the results of DuBois and those of Benedict, for the latter are incompatible with the theory.

[To be continued.] 